\title{
DINÂMICA HEMOCITÁRIA EM DIATRAEA SACCHARALIS F. (LEPIDOPTERA: CRAMBIDAE) DESAFIADA IMUNOLOGICAMENTE PELOS FUNGOS BEAUVERIA BASSIANA (BALS.) VUILL. E METARHIZIUM ANISOPLIAE (METSCH.) SOROK.
}

\author{
M.A.P. Oliveira ${ }^{1}$, V. Wanderley-Teixeira ${ }^{1}$, E.J. Marques ${ }^{1}$, \\ A.C. Albuquerque ${ }^{1}$, F.A.B. Santos ${ }^{3}$, R. Barros ${ }^{1}$, A.A.C.Teixeira ${ }^{2}$
}

${ }^{1}$ Universidade Federal Rural de Pernambuco, Departamento de Agronomia, Rua Dom Manoel de Medeiros, s/ $\mathrm{n}^{\circ}$, CEP 52171-900, Recife, PE, Brasil. E-mail: valeria@dmfa.ufrpe.br

\section{RESUMO}

\begin{abstract}
A pesquisa objetivou analisar, morfológica e quantitativamente, os tipos de hemócitos em lagartas do terceiro ínstar de Diatraea saccharalis F., desafiadas imunologicamente por Metarhizium anisopliae (Metsch.) Sorok. eBeauveria bassiana (Bals.) Vuill., nas concentrações $10^{3}, 10^{5} \mathrm{e} 10^{7}$ conídios $\mathrm{mL}^{-1}$, em comparação a lagartas não tratadas. Foram coletados $10 \mu \mathrm{L}$ de hemolinfa de cinco lagartas por tratamento, nos intervalos de 24, 36, 48, e 60 horas pós-inoculação, sendo contadas 300 células em campos aleatórios da lâmina, utilizando-se a objetiva de imersão (100X). Os hemócitos mais freqüentes foram os esferulócitos $(34,8 \%)$, plasmatócitos (29,7\%) e granulócitos (22,2\%), enquanto os menos freqüentes foram os prohemócitos $(8,3 \%)$, adipohemócitos $(2,6 \%)$ e oenocitóides $(2,4 \%)$. B. bassiana promoveu interferência efetiva na população de granulócitos na concentração de $10^{7}$ conídios $\mathrm{mL}^{-1}$ (24 e $36 \mathrm{~h}$ ) e plasmatócitos nas concentrações $10^{3}$ conídios $\mathrm{mL}^{-1}(60 \mathrm{~h})$ e $10^{7}$ conídios $\mathrm{mL}^{-1}$ (24 e 36h), onde ocorreu aumento do número de granulócitos e redução do número de plasmatócitos na maior concentração e, ainda, o aumento do número de plasmatócitos na concentração $10^{3}$ conídios $\mathrm{mL}^{-1}$. M. anisopliae não interferiu quantitativamente nos granulócitos e plasmatócitos. No que se refere aos esferulócitos, verificou-se maior tendência de redução do número dessas células no intervalo de $36 \mathrm{~h}$ para ambos os fungos, porém, diferença significativa só ocorreu na concentração de $10^{5}$ conídios $\mathrm{mL}^{-1}$ no intervalo de $60 \mathrm{~h}$, apresentando a menor média. Conclui-se que B. bassiana tem ação mais efetiva sobre a dinâmica populacional dos granulócitos e plasmatócitos em lagartas de $D$. saccharalis em relação ao M. anisopliae.
\end{abstract}

PALAVRAS-CHAVE: Hemócitos, fungos entomopatogênicos, morfologia, broca da cana-de-açúcar.

\section{ABSTRACT}

HEMOCYTIC DYNAMICS IN DIATRAEA SACCHARALIS F. (LEPIDOPTERA: CRAMBIDAE) IMMUNOLOGICALLY CHALLENGED BY BEAUVERIA BASSIANA (BALS.) VUILL. AND METARHIZIUM ANISOPLIAE (METSCH.) SOROK. FUNGI. This study was aimed to morphologically and quantitatively analyze the types of hemocytes inDiatraea saccharalis F.thirdinstar caterpillar immunologicaly challenged by Metarhizium anisopliae (Metsch.) Sorok. and Beauveria bassiana (Bals.) Vuill. in concentrations of $10^{3}, 10^{5}$ and $10^{7}$ conidia $\mathrm{mL}^{-1}$, compared to nontreated specimens. A $10 \mu \mathrm{L}$-sample of hemolymphs from five caterpillars for each treatment, in the intervals of $24,36,48$ and $60 \mathrm{~h} ; 300$ cells were counted in random fields of the lamina, using the immersion objective (100X). The most frequent hemocytes were the spherulocytes $(34.8 \%)$, plasmatocytes $(29.7 \%)$ and granulocytes $(22.2 \%)$, while the least frequent were the prohemocytes $(8.3 \%)$, the adipohemocytes $(2.6 \%)$ and the oenocytoids $(2.4 \%)$. B. bassiana promoted effective interference in the population of granulocytes at the concentration of $10^{7}$ conidia $\mathrm{mL}^{-1}(24$ and $36 \mathrm{~h})$ and of plasmatocytes at the concentrations of $10^{3}$ conidia mL $\mathrm{m}^{-1}(60 \mathrm{~h})$ and $10^{7}$ conidia $\mathrm{mL}^{-1}(24$ and $36 \mathrm{~h}$ ); there occurred an increase of the number of the granulocytes and a decrease of the number of plasmotocytes at the highest concentration, and an increase in the number of plasmatocytes at the concentration of $10^{3}$ conidia $\mathrm{mL}^{-1}$. M. anisopliae do not interfere quantitatively in the granulocytes and plasmatocytes. Regarding the spherulocytes, there was observed a higher tendency of

\footnotetext{
${ }^{2}$ Universidade Federal Rural de Pernambuco, Departamento de Morfologia e Fisiologia Animal, Área de Histologia, Recife, PE, Brasil.

${ }^{3}$ Universidade Federal de Pernambuco, FIOCRUZ, CPqAM, Recife, PE, Brasil.
} 
reduction in the number of these cells in the interval of 24 and $36 \mathrm{~h}$ for both fungi, but a meaningful difference only occurred at the concentration of $10^{5}$ conidia $\mathrm{mL}^{-1}$ in the $60 \mathrm{~h}$ interval, presenting the smallest average. It was possible to conclude that $B$. bassiana has a more effective action over the populational dynamics of the granulocytes and plasmatocytes of $D$. saccharalis caterpillars in association with $M$. anisopliae.

KEY WORDS: Hemocytes, entomopathogenic fungi, morphology, sugarcane borer.

\section{INTRODUÇÃO}

Os fungos entomopatogênicos são bastante conhecidos mundialmente por seu alto potencial no controle de diversas Ordens de insetos. De acordo com Alves; Pereira (1998), esses fungos causam distúrbios fisiológicos que atingem o tegumento e os sistemas, circulatório, reprodutor, respiratório, nervoso e digestivo dos insetos atacados.

Dentre os fungos entomopatogênicos, Metarhizium anisopliae (Metsch.) Sorok. e Beauveria bassiana (Bals.) Vuill. sãoutilizados como potentes agentes no controle biológico de insetos (Hegedus; Khachatourians 1996).

Nos insetos, os principais mecanismos de defesa são desempenhados pelos hemócitos, células livres circulantes na hemolinfa, cujo número e tipos diferem não só com a espécie estudada como também com a idade e desenvolvimento do indivíduo. Essas células fornecem uma resposta ágil e eficiente contra os patógenos que atingem a hemocele, participando ativamente dos mecanismos de defesa tais como: reconhecimento, fagocitose, encapsulação, coagulação, formação dos nódulos e citotoxicidade (GUPTA 1985).

Os hemócitos reconhecem uma variedade de corpos estranhos, pela interação direta de receptores de superfície celular com moléculas do organismo invasor, ou indiretamente pelo reconhecimento de receptores da resposta humoral que opsonizam a superfície do invasor (LAVINE; STRAND 2002). O sucesso das respostas de defesa depende do número e dos tipos de hemócitos envolvidos nestes mecanismos (RUSso et al., 2001).

Nosúltimosanos, tem havidoum enorme progresso no conhecimento das defesas imunológicas dos insetos (Hochetal., 2004; LeE et al., 2005). A biodiversidade desses organismos tem proporcionado modelos importantes para estudar as suas estratégicas antimicrobianas, as quais podem fornecer informações relevantes para o desenvolvimento de metodologias de controle biológico por patógeno. Nestesentido, sabe-se que as proteasesextracelulares produzidas por M. anisopliae e B. bassiana parecem participar na supressão da resposta imunológica celular na hemolinfa de larvas deGalleria mellonella L. (Lepidoptera: Noctuidae) (GRIESCH; VILCINSKAS 1998).

Alterações na morfologia, função e quantidade de hemócitos em insetos, ocasionadas por fungos entomopatogênicos, têm sido relatadas por vários pesquisadores. BalavenKatasubbaiah; Nataraju
(2005) verificaram susceptibilidade deBombyx moriL. (Lepidoptera: Bombycidae) a B. bassiana por este provocar uma interferência no número de alguns hemócitos com o progresso da infecção. Oprocesso de adesão dos hemócitos foi afetado em Manduca sexta L. (Lepidoptera: Sphingidae) após inoculação $\operatorname{com} B$. bassiana (DEAnA et al., 2004). Modificações na morfologia e ausência da atividade fagocitária dos plasmatócitos em G. mellonella quando inoculadas com M. anisopliae foram descritas por VILCINSKAs et al., (1997a; b).

Assim, o objetivo do presente trabalho foi analisar, morfológica equantitativamente, ostipos dehemócitos em lagartas do terceiro ínstar deDiatraea saccharalis $\mathrm{F}$. (Lepidoptera: Crambidae), submetidas aos fungos entomopatogênicos M. anisopliae e B. bassiana.

\section{MATERIAL E MÉTODOS}

Obtenção dos insetos: As lagartas deD.saccharalis foram obtidas da criação existente em laboratório.

Obtenção dos isolados de B. bassiana e $M$. anisopliae: Os isolados utilizados foram B. bassiana (447) obtido de Selenopsis invicta (Buren) eM. anisopliae (E9) obtido de Deois flavopicta (Stal.), provenientes da micoteca da Esalq, e mantidos no Laboratório de Patologia de Insetos a uma temperatura de $6 \pm 2^{\circ} \mathrm{Cem}$ tubos de vidro com meio de cultura Batata-DextroseÁgar, acrescido do antibiótico sulfato de estreptomicina (BDA+A) e óleo Nujol.

As suspensões dos fungos foram preparadas a partir de placas de Petri contendo meio de cultura (BDA + A) com os conídios, adicionando-se $20 \mathrm{~mL}$ de água destilada esterilizada para B. bassiana e $15 \mathrm{~mL}$ para $M$. anisopliae acrescido de espalhante adesivo Tween 80 a 0,01\% (ADE+E), sendo filtradas em gaze esterilizada e as concentrações para ambos os isolados foram aferidas em $10^{3}, 10^{5}$, e $10^{7}$ conídios $\mathrm{mL}^{-1}$, mediante o uso da câmara de Neubauer, conforme metodologia desenvolvida por Alves et al. (1998). A viabilidade de conídios foi avaliada por meio de plaqueamento em BDA+A, após 24h, em estufa incubadora BOD a $26 \pm 1^{\circ} \mathrm{C}$ e $12 \mathrm{~h}$ de fotofase, fazendo-se a contagem de conídios germinados e não germinados em microscópio de luz.

Tratamento tópico de D. saccharalis: As lagartas de terceiro ínstar de $D$. saccharalis foram pulverizadas com os fungos M. anisopliae e B. bassiana nas concen- 
trações de $10^{3}, 10^{5}$, e $10^{7}$ conídios $\mathrm{mL}^{-1}$, respectivamente. A testemunha foi pulverizada com água destilada esterilizada mais espalhante adesivo Tween 80, totalizando sete tratamentos.

Foram utilizadas 140 lagartas sendo 20 para cada tratamento, divididas em grupos de cinco indivíduos, a fim de permitir a coleta da hemolinfa, em diferentes intervalos de tempo $(24,36,48$ e 60 horas $)$, sendo cada lagarta considerada uma repetição. Estas foram acondicionadas em bandeja de plástico com compartimentos, contendo colmo de milho como substrato alimentar. A inoculação foi realizada através de pulverização com o auxílio de um microatomizador de marca Paasche "VL", sendo utilizado $1 \mathrm{~mL}$ da suspensão por tratamento. Em seguida, os insetos foram mantidos em condição de laboratório a uma temperatura de $27 \pm 2^{\circ} \mathrm{C}, 70 \pm 5 \%$ de UR e 12 horas de fotofase.

Coleta da hemolinfa para análise morfológica e contagem diferencial dos hemócitos: Amostras de 10 $\mu \mathrm{L}$ de hemolinfa foram coletadas de cincolagartas por tratamento, nos intervalos de 24,36 , 48, e 60 horas. Para isso realizou-se uma incisão na região mesopleural da lagarta e, com o auxílio de uma micropipeta, colheu-se a hemolinfa.

O material foi colocado sobre uma lâmina contendo $10 \mu \mathrm{L}$ de solução salina $(62 \mathrm{mM} \mathrm{NaCl}+100 \mathrm{mM}$ glicose + 10 mM EDTA + 30 mM citrato de sódio + 26 mM ácido cítrico + pH 4,6) para evitar a coagulação. Em seguida, procedeu-se o esfregaço, que foram mantidos por aproximadamente 20 minutos à temperatura ambiente para permitir uma melhor adesão dos hemócitos à lâmina. Oesfregaço foi fixado em metanol, por cinco minutos, sendo em seguida submetido à coloração pelo Giemsa, também por cinco minutos. A análise morfológica foi realizada utilizando microscópio deluz, da marca Olympus BX-49, efotografados em fotomicroscópio OLYMPUS BX-51. Para contagem diferencial dos hemócitos empregou-se metodologia descrita porFALLEIROset al. (2003), sendo contadas 300 células em campos aleatórios da lâmina, utilizando-se a objetiva de imersão (100X).

Análise estatística: A partir da contagem de 300 células por amostra, foi obtida a proporção de hemócitos (contagem diferencial) para cada tratamento e intervalo após tratamento. Assim, as porcentagens de cada tipo de hemócito foram submetidas à análise de variância (ANOVA) em esquema fatorial considerando 7 tratamentos e 4 intervalos de avaliação após tratamento como fatores principais. Havendo significância entre fatores principais, bem como de suas interações, ANOVA simples foi conduzida comparando as porcentagens entre tratamentos para cada intervalo de avaliação ou para cada tratamento em função dos respectivos intervalos de avaliação. Em seguida, quando foi caracterizada significância dos fatores pela ANOVA, as médias de tratamentos ou de intervalo de avaliação foram comparadas pelo teste de Tukey a 5\% de probabilidade. Todas as análises foram conduzidas empregando o programa estatístico SAS (SAs Institute, 2001).

\section{RESULTADOS E DISCUSSÃO}

As células mais freqüentes foram os esferulócitos, plasmatócitos e granulócitos, com percentuais de $34,8 \%, 29,7 \%$ e $22,2 \%$, respectivamente, enquanto que os hemócitos menos freqüentesforam os prohemócitos (8,3\%), adipohemócitos $(2,6 \%)$ e oenocitóides $(2,4 \%)$ (Fig. 1). Tal observação corrobora com os dados descritos por RAtcliffe (1993) e StRAND; Pech (1995), os quais relataram que nos estágios larvais em lepidópteros normalmente as células granulares e os plasmatócitos compreendem mais de $50 \%$ dos hemócitos circulantes, tendo a capacidade de adesão na superfície decorposestranhos.Osoutros hemócitos descritos em lepidópteros são células não adesivas, correspondendo aos esferulócitos, oenocitóides e prohemócitos.

Da mesma forma, FALLEIRoset al.(2003), trabalhando com microscopia de transmissão e varredura, também observaram maior freqüência de plasmatócitos, granulócitos e esferulócitos na hemolinfa de larvas de D. saccharalis. SegundoSAssetal.(1994), os esferulócitos estão envolvidos no transporte de componentes cuticular, o que pode explicar o seu maior percentual entre os hemócitos, pois as lagartas encontravam-se próximas à ecdise. Já os oenocitóides contêm precursores da fenoloxidase que provavelmente desempenha uma função na melanização (JIANG et al., 1997). Os prohemócitos são relatados como células-tronco que se diferenciam em um ou mais tipos de hemócitos.

Após inoculação com o fungo B. bassiana, diferenças significativas só ocorreram para os granulócitos na concentração de $10^{7}$ conídios $\mathrm{mL}^{-1}$ (24 e $36 \mathrm{~h}$ ) e para os plasmatócitos nas concentrações $10^{3}$ conídios $\mathrm{mL}^{-1}$ (60h) e $10^{7}$ conídios $\mathrm{mL}^{-1}$ (24 e 36h), onde houve um aumento do número de granulócitos e redução do número de plasmatócitos na maior concentração, e aumento do número de plasmatócitos na concentração $10^{3}$ conídios $\mathrm{mL}^{-1}$ (Tabela 1$)$.

O fungo M. anisopliae não interferiu significativamente nos granulócitos e plasmatócitos. No que se refere aos esferulócitos, verificou-se uma tendência de redução do número dessas células no intervalo de 24 e36h para ambos os fungos, porém, diferença significativa só ocorreu na concentração de $10^{5}$ conídios $\mathrm{mL}^{-1}$ no intervalo de $60 \mathrm{~h}$, resultando na menor média para esse tipo de hemócito quando desafiados pelo $M$. anisopliae. Não foram observadas alterações morfológicas nessas células após inoculação com os fungos entomopatogênicos em relação à testemunha. 


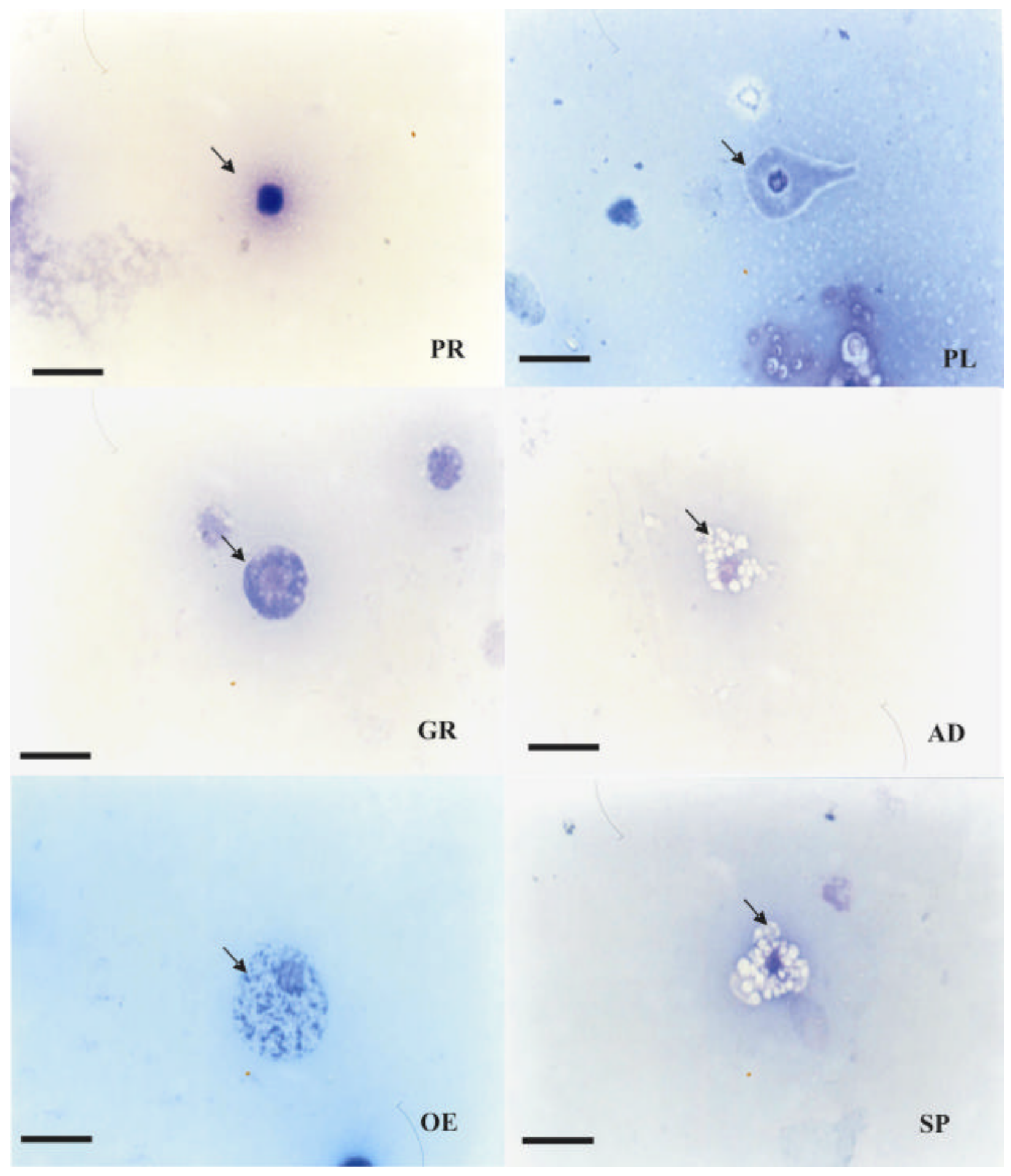

Fig. 1 - Tipos de hemócitos observados na hemolinfa de lagartas do terceiro ínstar de D. saccharalis: Prohemócito (PR), Plasmatócito (PL), Granulócito (GR), Adipohemócito (AD), Oenocitóide (OE) e Esferulócito (SP). Barras = $10 \mu \mathrm{m}$.

De acordo com Han et al. (1998), os hemócitos podem ser capazes de responder rapidamente às mudanças ambientais, devido às várias funções fisiológicas desempenhadas por essas células na hemolinfa, onde o número e proporção de cada tipo de hemócito liberado de órgãos hematopoéticos podem mudar rapidamente em respostas as várias reações imunes contra patógenos.

Os dados desta pesquisa mostraram uma pequena flutuação populacional dos hemócitos em respostas as diferentes concentrações dos fungosB. bassiana e M. anisopliae nos intervalos de $24,36,48$ e $60 \mathrm{~h}$. 
Apenas o fungo B. bassiana. na concentração $10^{7}$ conídios $\mathrm{mL}^{-1}$ nos intervalos de $24 \mathrm{e} 36 \mathrm{~h}$, demonstrou uma interferência mais efetiva na população dos granulócitos e plasmatócitos de D. saccharalis. Esses resultados estão de acordo com os obtidos por Hung; BOUCIAS (1996), os quais relataram um aumento do númerodegranulócitosem Spodopteraexigua (Hübner) (Lepidoptera: Noctuidae) e no gafanhoto Melanoplus sanguinipes F. (Orthoptera: Acrididae) inoculados com B. bassiana, enquanto que LeE et al. (2005) observaram uma diminuição do número de plasmatócitos em Mamestra brassicae L. (Lepidoptera: Noctuidae), quan- do inoculadas com Nomuraea rileyi (Farlow) Samson e B. bassiana.

Segundo Fuguet; Vey (2004), a redução dos plasmatócitos podeestar relacionada ao envolvimento dessas células no processo inicial de nodulação, enquanto que os granulócitos só participam dos estágios finais desse processo, o que justificaria o seu aumento. Esses mesmos autores sugerem ainda que o declínio do número de plasmatócitos em infecções com fungos pode estar relacionado à sua suscetibilidade aos metabólicos secundários tóxicos produzidos por estes.

Tabela 1 - Médias ( \pm EP) dos números de granulócitos (GR), plasmatócitos (PL) e esferulócitos (SP) da hemolinfa de lagartas do terceiro instar de D. saccharalis, desafiadas imunologicamente pelos fungos B. bassiana (B.b.) e M. anisopliae (M.a.).

\begin{tabular}{|c|c|c|c|c|c|}
\hline \multirow[t]{2}{*}{ Tratamento } & \multicolumn{4}{|c|}{ Tempo após tratamento (horas) } & \multirow{2}{*}{$\begin{array}{l}\text { Estatística }{ }^{1} \\
\mathrm{~F}_{3,17}{ }^{\mathrm{P}} \\
\end{array}$} \\
\hline & 24 & 36 & 48 & 60 & \\
\hline \multicolumn{6}{|l|}{ Granulócitos } \\
\hline Testemunha & $15,0 \pm 2,52 b^{2}$ & $19,7 \pm 4.05 \mathrm{~b}$ & $24,3 \pm 9,95$ & $30,7 \pm 2,73 a b$ & -3 \\
\hline B.b. $-10^{3}$ & $28,3 \pm 3,33 a b$ & $34,7 \pm 2,33 b$ & $37,7 \pm 6,36$ & $32,7 \pm 3,76 a b$ & -3 \\
\hline B.b. $-10^{5}$ & $29,3 \pm 2,90 \mathrm{ab}$ & $34,3 \pm 4,98 b$ & $34,3 \pm 8,35$ & $39,0 \pm 4,58 \mathrm{a}$ & -3 \\
\hline B.b. $-10^{7}$ & $44,3 \pm 8,68 \mathrm{aAB}$ & $61,7 \pm 9,17 \mathrm{aA}$ & $27,3 \pm 1,45 \mathrm{~B}$ & $31,3 \pm 4,10 \mathrm{abB}$ & $5,37^{0,0256}$ \\
\hline M.a. $-10^{3}$ & $23,7 \pm 2,96 \mathrm{bAB}$ & $33,0 \pm 2,00 \mathrm{bA}$ & $30,7 \pm 0,33 \mathrm{~B}$ & $20,7 \pm 2,73 \mathrm{bB}$ & $4,93^{0,0316}$ \\
\hline M.a. - $10^{5}$ & $31,7 \pm 0,88 \mathrm{ab}$ & $42,3 \pm 5,81 \mathrm{ab}$ & $29,7 \pm 4,63$ & $43,7 \pm 1,85$ a & -3 \\
\hline M.a. $-10^{7}$ & $26,0 \pm 0,58 \mathrm{abB}$ & $34,3 \pm 1,85 \mathrm{bAB}$ & $35,7 \pm 2,90 \mathrm{~A}$ & $39,7 \pm 1,45 \mathrm{aA}$ & $9,21^{0,0057}$ \\
\hline $\mathrm{F}_{6,14}{ }^{. P}$ & $4,96^{0,0064}$ & $6,60^{0,0018}$ & $-^{3}$ & $5,62^{0,0037}$ & \\
\hline \multicolumn{6}{|l|}{ Plasmatócitos } \\
\hline Testemunha & $36,0 \pm 3,46 a$ & $33,7 \pm 3,2 \mathrm{a}$ & $27,7 \pm 5,36$ & $21,7 \pm 4,25 b$ & -3 \\
\hline B.b. $-10^{3}$ & $32,7 \pm 6,12 \mathrm{a}$ & $29,0 \pm 4,58 \mathrm{ab}$ & $33,0 \pm 3,51$ & $38,3 \pm 3,18 a$ & -3 \\
\hline B.b. $-10^{5}$ & $35,3 \pm 3,48 a$ & $26,0 \pm 0,58 \mathrm{ab}$ & $30,7 \pm 2,60$ & $26,0 \pm 1,53 \mathrm{ab}$ & -3 \\
\hline B.b. $-10^{7}$ & $13,3 \pm 3,18 \mathrm{bB}$ & $13,7 \pm 3,84 \mathrm{bB}$ & $31,7 \pm 4,41 \mathrm{~A}$ & $27,0 \pm 4,58 \mathrm{abAB}$ & $4,92^{0,0318}$ \\
\hline M.a. - $10^{3}$ & $36,3 \pm 0,33 \mathrm{a}$ & $28,0 \pm 6,24 \mathrm{ab}$ & $28,7 \pm 4,48$ & $31,7 \pm 1,45 \mathrm{ab}$ & -3 \\
\hline M.a. $-10^{5}$ & $38,7 \pm 1,45$ a A & $27,7 \pm 1,67 \mathrm{abB}$ & $40,3 \pm 3,38 \mathrm{~A}$ & $35,0 \pm 3,21 \mathrm{abAB}$ & $5,23^{0,0273}$ \\
\hline M.a. $-10^{7}$ & $27,0 \pm 1,15 \mathrm{aB}$ & $33,0 \pm 1,00 \mathrm{aAB}$ & $32,7 \pm 1,76 \mathrm{AB}$ & $35,3 \pm 1,67 \mathrm{ab} A$ & $6,40^{0,0161}$ \\
\hline $\mathrm{F}_{6,14^{\prime}}{ }^{\mathrm{P}}$ & $8,23^{0,0006}$ & $3,84^{0,0179}$ & $-^{3}$ & $3,80^{0,0185}$ & \\
\hline \multicolumn{6}{|l|}{ Esferulócitos } \\
\hline Testemunha & $40,0 \pm 2,08$ & $34,7 \pm 8,76$ & $30,0 \pm 6,80$ & $34,3 \pm 6,43 a$ & -3 \\
\hline B.b. $-10^{3}$ & $31,0 \pm 4,58$ & $24,7 \pm 5,60$ & $24,3 \pm 2,18$ & $23,0 \pm 1,53 \mathrm{ab}$ & -3 \\
\hline B.b. $-10^{5}$ & $27,3 \pm 6,56$ & $29,0 \pm 3,00$ & $27,7 \pm 6,89$ & $27,0 \pm 5,13 a b$ & -3 \\
\hline B.b. $-10^{7}$ & $23,3 \pm 10,90$ & $14,7 \pm 4,05$ & $30,3 \pm 4,84$ & $24,7 \pm 5,90 \mathrm{ab}$ & -3 \\
\hline M.a. - $10^{3}$ & $33,0 \pm 3,51$ & $24,7 \pm 4,33$ & $30,3 \pm 7,36$ & $33,0 \pm 1,53 \mathrm{a}$ & -3 \\
\hline M.a. $-10^{5}$ & $23,7 \pm 2,33$ & $12,3 \pm 5,49$ & $20,0 \pm 1,53$ & $10,7 \pm 3,18 b$ & -3 \\
\hline M.a. $-10^{7}$ & $33,3 \pm 2,03$ & $27,7 \pm 1,20$ & $29,7 \pm 4,63$ & $21,0 \pm 2,52 \mathrm{ab}$ & -3 \\
\hline $\mathrm{F}_{6,14^{\prime}}{ }^{\mathrm{P}}$ & -3 & -3 & -3 & $4,22^{0,0124}$ & - \\
\hline
\end{tabular}


A menor interferência do fungo $M$. anisopliae na população dos granulócitos e plasmatócitos pode estar relacionada ao isolado e/ou concentração, pois, segundo SEWIFY; HASHEM (2001), quando G. mellonella foi inoculada com $M$. anisopliae, na concentração de $10^{7}$ conídios $\mathrm{mL}^{-1}$, ocorreu um aumento significativo do número de granulócitos. No entanto, GILLESPIE etal. (2000) observaram no gafanhoto Schistocerca gregaria (Forskal) (Orthoptera: Acrididae), uma redução significativa do número de granulócitos e plasmatócitos quando inoculados com M. anisopliae var. acridum, na concentração $7,5^{\prime} 10^{4}$ conídios $\mathrm{mL}^{-1}$.

\section{CONCLUSÃO}

A infecção de larvas de D. saccharalis com B.bassiana e $M$. anisopliae alterou a dinâmica populacional dos granulócitos e plasmócitos. No entanto, B. bassiana mostrou ação mais efetiva sobre esta dinâmica do que M. anisopliae. Não foi observado efeito significativo das concentrações de conídios na resposta hematocitária, sendo que apenas a maior concentração promoveu uma pequena alteração nesta resposta.

\section{AGRADECIMENTOS}

Aos pesquisadores Drs. Fábio André Brayner dos Santose Luiz Carlos Alves, Pesquisadores do CPqAMFIOCRUZ/UFPE- Recife, PE, pela contribuição científica para realização deste trabalhoeaoProf.Dr.JorgeBraz Torres, do Curso de Pós-Graduação em Entomologia Agrícola da UFRPE, pelas análises estatísticas.

\section{REFERÊNCIAS}

ALVES, S.B.; PEREIRA, R.M. Distúrbios fisiológicos provocados por entomopatógenos. In: ALVES, S.B. (Ed.) Controle microbiano de insetos. Piracicaba: FEALQ, 1998. p.39-54.

ALVES, S.B.; ALMEIDA, J.E.M; MOINOJUNIOR, A.; ALVES, L.F.A. Técnicas de laboratório. In: ALVES, S.B. (Ed.) Controle Microbiano de Insetos . Piracicaba: FEALQ, 1998. p.637-711.

BALAVENKATASUBBAIAH, M.; NATARAJU, B. Hemocyte changes during the progressive infection of Beauveria bassiana in different breeds of silkworm (Bombyx mori L.) Madras Agricultural Journal, v.92, n.7/ 9, p.431-437, 2005.

DEANA, P.; RICHARDSB, E.H.; EDWARDSB, J.P.; REYNOLDSA, S.E.; CHARNLEYA, K. Microbial infection causes the appearance of hemocytes with extreme spreading ability in monolayers of the tobacco hornworm Manduca sexta. Developmental and Comparative Immunology, v.28, n.7/8, p.689-700, 2004.
FALLEIROS, A.M.F.; BOMBONATO, M.T.S.; GREGÓRIO, E.A. Ultrastructural and quantitative studies of hemocytes in the sugarcane borer, Diatraea saccharalis (Lepidoptera: Pyralidae). Brazilian Archives of Biology and Technology, v.46, n.2, p.287-294, 2003.

FUGUET, R.; VEY, A. Comparative analysis of the production of insectisidal and melanizing macromolecules by strains of Beauveria spp. in vivo studies. Journal of Invertebrate Pathology, v.85, n.3, p.152167, 2004.

GILLESPIE, J.P.; BURNETT, C.; CHARNLEY, A.K. The immune response of the desert locust Schistocerca gregaria during mycosis of the entomopathogenic fungus, Metarhizium anisopliae var acridum. Journal of Insect Physiology, v.46, n.4, p.429-437, 2000.

GRIESCH, J.; VILCINSKAS, A. Proteases released by entomopathogenic fungi impair phagocytic activity, attachment and spreading of plasmatocytes isolated from haemolymph of the greater wax moth, Galleria mellonella. Biocontrol Science Technology, v.8, n.4, p.517531, 1998.

GUPTA, A.P. Cellular elements in hemolynph. In: KERKUT, G.A.; GILBERT, L.I. (Ed.). Comprehensive insect physiology, biochemistry and phamacology. Oxford: Pergamon Press, 1985. v. 3, p. 401-451.

HAN, S.S.; LEE, M.H.; KIM, W.K. Hemocytic differentiation in hemopoietic organ of Bombix mori larvae. Zoological Science, v.15, n.3, p.371-379, 1998.

HEGEDUS, D.D.; KHACHATOURIANS, G.G. Analysis of cellular defense reactions of the migratory grasshopper, Melanoplus sanguinipes, infected with heat-sensitive mutants of Beauveria bassiana. Journal Invertebrate Pathology, v.68, n.2, p.166-169, 1996.

HOCH, G.; SOLTER, L.F; SCHOPF, A. Hemolymph melanization and alterations in hemocyte numbers in Lymantria dispar larvae following infections with different entomopathogenic microsporidia.

Entomologia Experimentalis et Applicata, v.113, n.2, p.7786, 2004.

HUNG, S.H.; BOUCIAS, D.G. Phenoloxidase activity in hemolymph of naïve and Beauveria bassiana infected Spodoptera exigua larvae. Journal Invertebrate Pathology, v.67, n.1, p.35-40, 1996.

JIANG, H.; WANG, Y.; MA, C.; KANOST, M.R. Subunit composition of pro-phenol oxidase from Manduca sexta: molecular cloning of subunit PPO-pl. Insect Biochemistry and Molecular Biology, v.27, n.10, p.835-850, 1997.

LAVINE, M.D.; STRAND, M.R. Insect hemocytes and their role in immunity. Insect Biochemistry and Molecular Biology, v.32, n.10, p.1295-1309, 2002. 
LEE, M.; YOON, C.S.I.K.; YI, J.I.; CHO, J.R.; KIM. H.S. Cellular immune responses and FAD-glucose dehydrogenase activity of Mamestra brassicae (Lepidoptera: Noctuidae) challenged with three species of entomopathogenic fungi. Physiological Entomology, v.30, n.3, p.287-292, 2005.

RATCLIFFE, N.A. Cellular defense responses of insects: unresolved problems. In: BECKAGE, N.E; THOMPSON, S.N.; FEDERICI, B.A. (Ed.). Parasites and Pathogens of Insects. San Diego: Academic Press, 1993. v.1, p.267-303.

RUSSO, J.; BREHÈLIN, M.; CARTON, Y. Haemocyte changes in resistant and susceptible strains of Drosophila melanogaster caused by virulent and avirulent strains of the parasitic wasp Leptopilina boulardi. Journal of Insect Physiology, v.47, n.2, p.167-172, 2001.

SAS INSTITUTE. SAS/STAT User's guide, version 8.2, TS level 2MO. SAS Institute. Inc., Cary, N.C., 2001.

SASS, M.; KISS, A.; LOCKE, M. Integument and hemocyte peptides. Journal of Insect Physiology, v.40, n.5, p.407-421, 1994.

SEWIFY, G.H.; HASHEM, M.Y. Effect of the entomopathogenic fungus Metarhizium anisopliae
(Metsch.) Sorokin on cellular defense response and oxygen uptake of the wax moth Galleria mellonella L. (Lep., Pyralidae). Journal of Applied Entomology, v.125, n.9/10, p.533-536, 2001.

STRAND, M.R.; PECH, L.L. Immunological basis for compatibility in parasitoid-host relationships. Annual Review of Entomology, v.40, p.31-56, 1995.

VILCINSKAS, A.; MATHA, V.; GÖTZ, P. Effects of the entomopathogenic fungus Metarhizium anisopliae and its secondary metabolites on morphology and cytoskeleton of plasmatocytes isolated from the greater wax moth, Galleria mellonella. Journal of Insect Physiology, v.43, n.12, p.1149-1159, 1997a.

VILCINSKAS, A.; MATHA, V.; GÖTZ, P. Inhibition of phagocytic activity of plasmatocytes isolated from Galleria mellonella by entomogenous fungi and their secondary metabolites. Journal of Insect Physiology, v.43, n.5, p.415-483, 1997b.

Recebido em $7 / 3 / 07$

Aceito em 19/5/08 